\title{
Über die Straufsenzucht.
}

\section{Von W. Bassermann.}

Glatz, Donjon im August 1910.

In die Reihe unserer Haustiere kann als jüngstes der Straufs, Struthio australis eingegliedert werden. Sein Produkt, die Straufsenfeder war schon zu allen Zeiten hochgeschätzt. Seit Menschengedenken sehen wir diese selten schönen Federn bald als Kriegs-, bald als Festschmuck; die Könige der Ägypter, die vornehmen Römerinnen, die Ritter, Sänger und Frauen des Mittelalters, die Hö̈linge Englands und Frankreichs und heute die Vertreterinnen aller Kulturländer - von allen wird die Straufsenfeder als eine vornehmliche Zier der Vornehmen betrachtet und wegen ihrer Seltenheit und Schönheit hoch bewertet. Die Nachfrage des Weltmarktes nach Straufsenfedern wuchs mit der zuuehmenden Wohlhabenheit der Völker. Die verhältnismälsig einfache Art, durch Jagd auf die Straufsen die Federn zu erwerben genügte zunächst, den Federnkonsum zufriedenzustellen. Genügte durch Jahrhunderte hindurch, bis in neueren Zeiten durch die vermehrte Nachfrage Konsum und Produktion in ein Milsverhältnis gerieten. Die technischen Verbesserungen der Jagdutensilien hielten zwar dem Zurückziehen der Straulsen in unwegsame Wüstenstriche einigermalsen das Gleichgewicht. Die unverhältnismäfsig sich mehrende Nachfrage resultierte in exorbitanten Preisen, die wiederum grofse und kostspielige Jagdexpeditionen rentabel machten. Ruhelos und gehetzt, im Brutgeschäft gestört, verbannt in futterlose Gegenden mufste der Straufs in Riesenschritten seiner vollkommenen Vernichtung entgegengehen. Menschliches Empfinden und Geschäftsklugheit verboten, dieser Ausrottung müfsig zuzusehen. Ein französischer Privatmann, A. Chagot, regte um die Mitte des 19. Jahrhunderts als erster eine systematische Zucht dieses Vogels an, welche an Stelle der vernichtenden Jagd dasselbe Ziel in Aussicht stellte. Die Hauptschwierigkeit sah man in der scheinbaren Unmöglichkeit einer Reproduktion in der Gefangenschaft. Chagot schrieb Preise aus und machte selbst umfangreiche Versuche, mit geringem Erfolg. 1859 nahm sich die Societé d'Acclimatisation der Frage an und setzte einen erbeblichen Geldpreis aus "für die erfolgreiche Domesticierung des Straufsen in Algier, am Senegal oder in Europa“. Den ersten Beweis, eine gesunde Nachzucht von Straufsen in der Gefangenschaft erzielt zu haben brachte Hardy, der Direktor des Acclimatisationsgartens in Hanna in Algier. Seine Nachfolger und andere Interessenten stellten die Tatsache fest, wirtschaftlicher Basis aufgebaut, gute Resultate liefern könne. Doch beschränkte man sich in Algier auf Versuche, eine Betätigung der Landwirte auf diesem Gebiete blieb aus. Den Engländern 
war es überlassen, diese praktisch erprobte Theorie in einer gesunden, grolszügig durchgeführten und vor allem äufserst gewinnbringenden Praxis in Südafrika auszubauen. Die Haltung von Straufsen war den südafrikanischen Farmern nicht vollkommen fremd gewesen, in kleinem Malsstabe wurden wildgefangene Straufsen auf den Farmen gehalten, doch Zuchtversuche hatte noch njemand angestellt. Nach authentischen Mitteilungen wurden 1866 die ersten Straulsenzuchten in Beaufort West und Oudtshorn in der Kapkolonie eingerichtet. 1870 fanden die erfolgreichen Anfänge Nachahmer im Georgedistrikt, und von diesem Zeitpunkt an nahm der neue Zweig afrikanischen Landwirtsehaftsbetriebes seinen Siegeszug durch das ganze Land. Die Straufsenzucht erfuhr einen derartigen Aufschwung, wie er noch in keinem landwirtschaftlichen Betrieb beobachtet werden konnte und nur einigermalsen durch die Umwälzungen in der Wollschafzucht durch Merinozüchtung erreicht wird. So erwähnt der Census über Viehzucht in Südafrika im Jahre 1865 nur 80 zahme Straufse. 1875 er. schienen unter dieser Rubrik schon 32247, und bis 1880 war der Bestand auf 150000 angewachsen. Diese rapide Steigerung wurde mit mäfsigen, teils durch zeitweise Überproduktion, teils durch den verheerenden Krieg verursachten Schwankungen bis beute beibehalten, sodafs Prof. Duerden, wohl unser berufenster Straufsenforscher, mir seine heutige Schätzung auf 1000000 zahmer Straufsen angibt.

Dieser ungewöhnliche Aufschwung der Straufsenzucht weist derartige Dimensionen auf, dafs die Vermutung nabe liegt, als ob dieser Betrieb ohne irgend weiche Schwierigkeiten und Rückschläge zu bewerkstelligen wäre. Doch ist dies keineswegs der Fall, nähere Beschäftigung mit diesem Thema wird sogar das Gegenteil ergeben. Der Grund dieser Blüte wird daher darin zu suchen sein, dais die relativ hohen Preise, welche die Federn auf dem europäischen Markte erzielten, den Straufsenfarmern die Möglichkeit boten, auch bei unrationeller Wirtschaft, bei grolser Sterblichkeit unter den Zuchttieren und bei nur geringer Ausnutzung der gegebenen Produktionsbedingungen das im Straulsenbetrieb investierte Kapital günstig zu verzinsen. Das stetig sich mehrende Angebot eilte der immerhin steigenden Nachfrage im Laufe der Jahre voraus, so dafs eine Wertrerminderung der Federn um ca. $40 \%$ erfolgte. Als natürliche Schlu[sfolgerung ergab sich daraus für die Farmleiter die Notwendigkeit, die vorhandenen und durch Kapitalanlage geschaffenen Möglichkeiten eindringlicher auszunutzen, d. h. rationeller zu wirtschaften. Dieses Bestreben, unterstützt durch die Erfahrungen in der Zucht, macht sich auch in der, die Straulsenzucht während der ersten Dekaden ihres Bestehens stark vernachlässigenden wissenschaftlichen Forschung bemerkbar. Während die wenigen Bücber, welche dieser Frage gewidmet waren, sich meist darauf beschränkten, referierend, tatsächlich bestehende Betriebe zu schildern, im übrigen sehr 
wertvolle, jedoch meist laienhafte Beobachtungen über die Natur des Straufsen vorzubringen, haben erst die letzten Jahre einen merklichen Fortschritt in der Kenntnis der eine rationelle Straufsenzucht bedingenden Faktoren an der Hand wissenschaftlicher Versuche gezeitigt. Diese Forschungen, in Verbindung mit den sich häufenden praktischen Erfahrungen der Züchter haben diejenigen Forderungen, welche die Erzielung eines fehlerfreien, hochwertigen Endproduktes an die Betriebsmethode stellt, mehr und mehr präzisiert, und es soll die Aufgabe dieser Arbeit sein, den Stand der Straufsenzucht, wie er sich auf Grund der heutigen Forschungsergebnisse herausgebildet bat, näher zu beleuchten.

Der Straufsenfarmbetrieb verfolgt $z$ wei Ziele:

1. Die Vermehrung des Herdenbestandes durch natürliche Fortpflanzung.

2. Die Erzielung der höchsten Marktfähigkeit der Produkte der ausgewachsenen Straufse, die Federngewinnung.

Naturgemäls hatte sich dementsprechend die Wissenschaft hauptsächlich mit diesen Fragen zu beschäftigen. Es war zu eruieren, welche Haltungs- und Ernäbrungsbedingungen für den Straufsen geschaffen werden mufsten, um die Tiere selbst in vollstem Gesundheitszustand zu erhalten und dadurch hochbewertete Produkte: eine gesunde Nachzucht und fehlerfreie Federn zu erzielen.

Grofse Schwierigkeiten bereitet hierbei die aufserordentliche Empfindlichkeit des Straufsen gegen äufsere Einflüsse, deren Einwirkung auf die Qualität der Produkte durch den komplizierten Bau der Feder in hohem Mafse verstärkt wird. Die Feder stellt ein Gebilde der Hautzellen dar, welches durch vermehrten Blutandrang zu den Zellen der Epidermis und der Malpighischen Schicht hervorgerufen wird. Aus einer, Federkeim genannten Papille bildet sich durch Vermehrung der Blutgefäfse in Verbindung mit reichen Lymphgeweben die sogenannte Pulpa, der eigentliche Nährboden der Feder. Sie ordnet die, ihr zunächst liegenden Zellen zu radiär gelagerten, keilförmigen Gruppen an, die sternartig in die Pulpa eindringen und deren jede beim Aufbau der Feder ihre bestimmte Funktion übernimmt. Prof. Duerden in Grahamstown war der erste, der einiges Licht in den inneren Zusammenhang zwischen Blutzirkulation und Federnbildung brachte. Er wies nach, dals nur ein Individuum, das in bester Kondition, sich im Vollbesitz seiner Kraft befindet, eine erstklassige Feder zu produzieren imstande ist, sodafs jede, noch so geringe Schwankung im Gesundbeitszustand des Produzenten, Defekte an der Feder zeitigen mufs. Jede Krankheit resultiert in einer gewissen Unregelmälsigkeit der Blutzirkulation, der Blutdruck in den äufsersten, die Feder ernährenden Kapillaren wird dementsprechend schwanken - der Bau der Feder infolgedessen ungleichmälsig sein. Der Farmbetrieb mufs daher 
sein Schwergewicht auf die Schaffung derjenigen Bedingungen legen, die es ermöglichen, Krankheiten und Verschlechterung der Kondition nach Kräften zu vermeiden.

Einen weiteren Faktor bildet der Umstand, dafs schon in den ersten Entwickelungsstadien der Charakter der Feder fixiert wird. Befindet sich der Vogel zu der Zeit, in welcher die Pulpa zu neuem Wachstum angeregt wird, in mangelhafter Kondition, sei es durch Krankheit oder schlechte Ernährungsverhältnisse, so wird die sich entwickelnde Feder schlecht und fehlerhaft bleiben, auch wenn für die Verbesserung der Kondition des Tieres Sorge getragen wird. Es mufs daher Wert darauf gelegt werden, das in den Tagen, in welchen der Kiel der alten Feder entfernt und hierdurch die Pulpa veranlalst wird mit der Bildung der neuen Feder zu beginnen, der Strauls sich in einwandsfreiem Gesundheits- und Ernährungszustand befindet.

Der Jahresumtrieb auf einer Straufsenfarm wird des Weiteren durch die Zeitdaner beeinflufst, welche eine Feder zur völligen Reife benötigt. Unter relativ günstigen Bedingungen kann angenommen werden, dafs von dem Augenblick, in welchem die Pulpa mit der Neubildung beginnt, bis zu dem Zeitpunkte, in welchem sie ihre volle Entwicklung und grölste Schönheit erreicht hat, ein Zeitraum von 6 Monaten verstreicht. Obgleich in diesem Stadium die Feder noch nicht abgestorben ist, die Pulpa vielmehr mit blutführenden Kapillaren noch ein erbebliches Stück in den Kiel hineinreicht, ist es angemessen, dann schon die Feder zu ernten, da sie, am Vogel belassen, durch äufsere, mechanische Einwirkungen zu leicht gefährdet wird. Sie wird im oberen Drittel der Spule, an einer Stelle kurz unter den untersten rami, wo also die Pulpa nicht mehr verletzt werden kann, abgeschnitten. Das in der Follikel zurückbleibende Spulenende benötigt wiederum 2 Monate, um rollends auszutrocknen, wo es ohne Gefahr ausgezogen werden kann, um die Pulpa zu neuem Wachstum anzuregen. Von Entspulen zu Entspulen mufs daher ein Zeitraum von mindestens 8 Monaten verstreichen, was drei Ernten in zwei Jahren bedeutet. Ursachen der versehiedensten Art können jedoch das Wachstum einzelner Federn hemmen, so dafs Schwankungen von 2-3 Wochen beobachtet werden. Prof. Duerden stellte vielfache Experimente an, um die, das Federnwachstum beeinflussenden physiologischen Faktoren zu fixieren und kam zu dem Resultate, dals schon schwaches Kränkeln des Tieres das Tageswachstum der Feder von $5 \mathrm{~mm}$ auf $3,5 \mathrm{~mm}$ zu reduzieren vermag; bei schwerer Krankheit das Wachstum eine Woche und länger völlig onterbleibt. Während die Pulpa unter gewöhnlichen Bedingungen durch Belassen des Spulenendes in der Federfollikel in Ruhezustand gehalten werden kann, ist dies bei besonders günstigen Ernährungsverhältnissen nicht möglich. Ist bei vorteilhaftem Klima eine Trockenheitsruhepause der Vegetation nur gering ausgeprägt, so wird die 
Pulpa ein derart reges Wachstum zeigen, dafs sie, nachdem sie sich völlig aus der alten Spule zurückgezogen hat, also nach acht Monaten mit der Bildung der neuen Feder beginnt, deren Spitze durch die in der Follikel steckende Spule am Austreten gehindert und dadurch verletzt werden kann. Doch trifft dieser Umstand nur in wenigen Gegenden zu, vielmehr wird meist durch Belassen der Spule am Vogel, resp. Entspulen der Zeitpunkt der Neubildung einer Feder willkürlich bestimmt werden können. Es bestehen dementsprechend zwei Umtriebe auf den Straufsenfarmen, je nach den klimatischen und vegetativen Vorbedingungen, deren einer eine Ernte alle acht, der andere alle zwölf Monate ermöglicht.

Der Achtmonate-Umtrieb ist nur unter günstigen Verhältnissen rentabel und verlangt eine weitgehende Emanzipierung von den klimatischen Einflüssen. In rationellen Durchschnittsbetrieben wird daher der Jahresturnus vorzuziehen sein. Hierbei ist Gelegenheit gegeben, folgende natürlicben Faktoren nutzbringend $\mathrm{zu}$ verwerten. $\mathrm{Da}$ die Federn, verkümmert durch Nichtgebrauch, lediglich als sekundäre Geschlechtscharaktere anzusprechen sind, stehen sie in ihrer Entwicklung in engen Beziehungen zu den sexuellen Funktionen. Es ist ein wissenschaftlich bewiesener Erfahrungssatz, dafs die Feder zu Beginn der Brunst- und Paarungsperiode ihre grölste Schönheit erreicht hat. Es ist daher empfehlenswert, zu diesem Zeitpunkte die Ernte zu bewerkstelligen. Der vermehrte Stoffverbrauch einer ergiebigen sexuellen Tätigkeit würde jedoch der Pulpa eine grofse Anzahl zum Aufbau der Feder notwendigen Stoffe entziehen, so dafs es wichtig ist, mit dem Entspulen so lange zu warten, bis eine Periode grölserer Ruhe die Tiere in verbesserte Kondition gebracht hat. $\mathrm{Da}$ die Paarungszeit im Mai beginnt und im November zu Ende geht, kann man gleichzeitig das Entspulen und hierdurch die Neubildung der Feder in eine Zeit verlegen, zu welcher, nach den ersten Regenschauern die Weide mit frischem Grün überzogen ist und durch Güte und Nährstoffgebalt des Futters eine gute Kondition des Vogels ermöglicht wird. Es ist also ein gewisser Spielraum gelassen, innerhalb dessen der Zeitpunkt, zu welchem die Ernte zu erfolgen hat, gewählt werden kann. Eine Ausnahme hiervon bildet das Schneiden der Erstlingsfeder, welches unter allen Umständen nach dem sechsten Monat zu erfolgen hat. Die auf die Erstlingsfeder folgende Jugendfeder zeigt ein derart reges Wachstum, dafs sie sich mit ihrer Spitze in die Spule der Erstlingsfeder hineindrängt, falls diese nicht zur rechten Zeit, nach acht Monaten, entfernt wird. Da jedoch nicht alle Keime der Jugendfeder gleichmälsig die Erstlingsfeder zu verdrängen suchen, wird durch allzulanges Belassen der Erstlingsfederspule in der Follikel ein ungleichmäfsiges Wachstum der Federn eines Vogels eingeleitet, das später nur mit grörsten Schwierigkeiten reguliert werden 
kann. Das Entspulen hat mit grofser Vorsicht zu geschehen. Wird die Spule zu früh entfernt, so kann eine Verletzung der Pulpa verursacht werden, die ein Verkümmern oder völliges Fehlen der aus diesem Keime zu bildenden späteren Feder bewirkt.

Durch kluge Zuchtwahl kann auf die Erzielung vollendet guter Federn günstig eingewirkt werden. Während bei anderen landwirtschaftlichen Betrieben die lange Erfahrung gewisse Gesichtspunkte gezeitigt hat, wobei aus äufseren Merkmalen auf die, einem Tiere innewohnende Tendenz, ein bestimmtes Produkt in hervorragender Qualität oder Quantität zu erzeugen, geschlossen werden kann, ist die systematische Straufsenzucht noch zu jung, als dafs ein Idealtypus sich bätte bilden lassen. Der Züchter mufs daher die Tiere individuell beobachten und nur solches Material zur Zucht verwenden, welches bei robuster Gesu ndheit gute, einwandsfreie Federn liefert. Kreuzungen zwischen dem in BritischSüdafrika gezüchteten Str. australis und dem nordafrikanischen Str. camelus haben zeitweise gute Erfolge gebracht. Ein Vogel, der nicht in jeder Beziehung den Ansprüchen an ein vollkommenes Zuchttier genügt, sollte an der Paarung verhindert werden. Das in Südafrika angelegte „Ostrich-Studbook" ist vorzüglich geeignet, solche Bestrebungen $\mathrm{zu}$ unterstützen. Mit Pédigree versehene Exemplare werden häufig zum zehnfachen Preise eines gewöhnlichen Zuchtstraufsen verkauft. Tiere, die aus irgend einem Grunde zur Zucht nicht zugelassen werden sollten, müssen entweder in eigenen Triften gehalten oder kastriert werden. Das Kastrieren männlicher und weiblicher Tiere verursacht nach der Elley'schen Methode auch bei der Ausübung durch Laien nur geringe Schwierigkeiten und der Prozentsatz an tödlichem Ausgang dieser Operation ist nicht gröfser als bei anderen Haustieren. Die Entfernung der Reproduktionsorgane wirkt auch in anderer Hinsicht günstig auf die Federnproduktion ein, indem sich bei der nunmehr sehr bedächtigen Lebenweise auch nervöse und in ihrer Erregbarkeit schwer in guten Ernährungszustand zu bringende Tiere aufserordentlich rasch und vollständig erholen.

Der Einflufs der Ernährung auf die Federnbildung ist noch nicht in vollem Umfang aufgeklärt. Nacbgewiesen ist, dafs bei einseitiger Ernährung durch Luzerne schwerere und weniger elastische Federn erzielt werden. Inwiefern jedoch ein Produktionsfutter eine Einwirkung auf die Federnbildung ausüben kann und welche Zusammensetzung ein solcher Nährstoffüberschufs aufweisen mufs um ein günstiges Resultat zu erzielen, ist eine vorläufig noch offene Frage. Bei dem grofsen Ausschlag, den Ernährungsschwankungen aut die Art und die Menge des Produktes ausübt, mürste die empirisch erzielte Ernährungsweise durch eine wissenschaftlich exakte Fütterungsmethode ergänzt werden. In der Mehrzahl der Betriebe wird man zwar noch auf 
Jahre hinaus von einer derart rationellen Fütterung absehen müssen, da meist die Art der Straufsenhaltung eine solch intensive Wirtschaftsweise noch nicht zulälst. Die anspruchsvoller werdende Nachfrage des Federmarktes wird Zugeständnisse von Seiteu der Züchter verlangen, die infolgedessen mehr und mehr Wert auf gutes Zuchtmaterial legen müssen, wodurch in grölserem Umfange als bisher specifische Qualitätszuchten bervorgerufen werden, deren Nachzucht zur Blutauffrischung in extensiven Betrieben dienen mufs. Eine solche rationelle Qualitätszucht ist aber ohne genaue Kenntnis der Ernährungsphysiologie schwer durchzuführen. Vorläufig wird in arbeitsextensiven Betrieben zur Ernährung der Straufse die heimiscbe Vegetation genügen. Hierbei sind Caroo, Süls-, Sauergrasfarmen zu unterscheiden. Caroo stellt eine Mischung von stauden- und krautartigen Pfianzen mit den alkalireichen Chenopodeenformen und vielen Arten Gramineen dar, während bei Süfs- und Sauergrasgegenden das nährstoffreiche Element niederer Büsche stark zurückgedrängt wird. Bei intensiveren Betrieben tritt mit wachsender Intensität eine vermehrte künstliche Ernährung an Stelle der natürlichen Weide. Luzerne wird den Straufsen sowohl als Häcksel wie als Grünfutter und Weide zugängig gemacht. Zur Zeit der Brunst und des Eierlegens muls eine Körnerration verabfolgt werden, wodurch in gewissem Mafse die Anzahl befruchteter Eier vermehrt werden kann. Bei mangelndem Salzgehalt der natürlichen Vegetation mufs das fehlende $\mathrm{P}_{2} \mathrm{O}_{5}$ dessen der Straufs eine grofse Menge bedarf, durch Knochenmehl oder gehackte Knochen ersetzt werden. Die Nahrungszufuhr mufs reichlich sein um dem Tiere die nötigen Baust.offe zuzufübren, doch mufs ein allzustark reizendes Futter vermieden werden, da es die Nervosität der Tiere vermehrt und einem guten Gesundheitszustand hinderlich ist.

Bei der starken Empfindlichkeit der Straufsen gegen jegliche Schwankungen in ibrer Haltung sind sie vielerlei Krankheiten sowohl konstitutioneller als infektiöser Natur ausgesetzt. Die Federnproduktion wird nur mittelbar durch solche Erkrankungen des Vogelorganismus beeinträchtigt und resultiert fast stets in einem als Schnabelhiebigkeit (Bar) bekannten Defekte der rami und radii. Auch hier war Duerden der erste, der die Art der Bar-Bildung einwandsfrei nachwies. Seine Theorie, die zweifellos dem tatsächlichen Vorgang entspricht, baut sich auf folgenden Beobachtungen auf. Auch unter günstigsten Bedingungen gebt das Wachstum der Feder nicht in vollkommener Gleichmärsigkeit vor sich sondern wechselt in der Ausbildung der einzelnen Teile je nach der Stärke des zu der Pulpa strömenden Blutzuflusses. Regelmäfsige Schwankungen im Blutandrang resultieren aus der wechselnden Intensität der physiologischen Umsetzung bei Tag und bei Nacht. Die kühle Nachttemperatur zusammen mit verminderter Stoffwechseltätigkeit setzt den Blutdruck in den Capillaren herab, so dals ein schwächeres Nachtwachstum im Gegen- 
satz zu dem stärkeren Tageswachstum zu konstatieren ist. Tritt gleichzeitig mit der schwächeren Ausbildung der einzelnen Federnteile während des Nachtwachstums eine anormale Verminderung des Blutdruckes in der Pulpa auf, wie es durch krankhafte Störungen der Stoffwechseltätigkeit hervorgerufen werden kann, so wird infolge des vermehrten Druckes, mit welchem die umgebenden Hautzellen auf die noch weiche, nur wenig verhornte Feder eiuwirken, an den von Anfang an schwächer ausgebildeten Stellen der Feder eine Schrumpfung hervorgerufen. Diese Schrumfung wird die Bildung der rami und radii völlig hemmen, so dals beim Austreten der Feder aus der Oberhautschicht und ihrem Entfalten der Defekt sich in Form eines Striches über sãmtliche Strahlen quer über die Fahne hinziehen wird. Schnabelhiebigkeit entwertet die Feder in hohem Mafse, besonders da die Strahlen an der Stelle des Bars leicht knicken und der Feder ein unschönes Aussehen geben. Neben der Schnabelhiebigkeit treten noch andere Federdefekte auf, die sich bald in ungleichm äIsiger Entwicklung der Fahne, bald in vôlligem Fehlen oder unregelmäfsigem Stand der Äste äufsern. Wenn diese Fehler auch zum Teil auf mechanische Einwirknngen zurückgeführt werden können, liegt doch meist die Ursache in physiologischen Einwirkungen, welche durch Krankheitserscheinungen irgend welcher Art hervorgerufen werden. Áhnlich den anderen, die Straulsen betreffenden Fragen beschäftigte sich die Wissenschaft - in letzter Zeit auch eingehend mit der Pathologie dieser Vögel. Doch sind auch auf diesem Gebiete noch manche Aufgaben zu lösen. Von grofsem Einflusse auf die Blutzirkulation und infolgedessen indirekt auf die Federnbildung sind die beim Straulsen äufserst häufig auftretenden Verdauungsstörungen. Eine rationelle Haltung und Fütterung wird in dieser Hinsicht in vielen Fällen Abhilfe schaffen, es sei denn, dafs die mangelhafte Ausnutzung der Nährstoffe eine Folgeerscheinung der, nur allzuoft auftretenden Infektion der Verdauungswege durch Würmer darstellt. Hierbei kommen bautsächlich zwei Arten in Betracht: Taenia struthionis, der Straufsenbandwurm und Strongylus Douglassii der sogenannte Drahtwurm.

T. struthionis gehört zur Gattung der Cestoden und hält sich, als geschlechtsreife Form der Plattwürmer, als Bandwurm im Darm des Straufsen auf. Welches Tier die T. struthionis als Zwischenwirt aufsucht konnte bisher noch nicht festgestellt werden. Prophylaktische Mafsregeln sind daher äufsert schwierig und von ungewissem Erfolg. Bei der Häufigkeit des Vorkommens der Taenia ist es ratsam, sämtliche Vögel der Herde alle sechs Monate, ob sie Symptome zeigen oder nicht, mit abtreibenden Mitteln zu behandeln. Als Medizin wird Petroleum angewandt, und 2war je nach dem Alter des Patienten $1 / 4$ bis $1 / 21$. pro Kopf auf nüchternen Magen. Gute Erfolge zeitigte auch die Verwendung des Kamalapulvers, welches aus den Drüsen und 
Körnchen von Mallotus philippinensis besteht und ein spezifisches Abführmittell darstellt.

Weitaus gefährlicher, weil häufig von tödlichem Ausgange gefolgt, ist das Auftreten des Strongylus Douglassii. Über die Art, die Ernährung, Fortpflanzung und Infektionsmethode dieses Parasiten existieren noch keine exakten Forschungen. Str. douglassii, ein Pallisadenwurm, ist $8-10 \mathrm{~mm}$ lang, von durchsichtigem Weifs, erscheint jedoch rötlich auf der entzündeten Magenschleimhaut. Er hält sich allein im Drüsenmagen auf, wo er sich auf die obere, reich mit Drüsen und Drüsenausgängen versehene Magenwandung aufheftet. Da seine Oberfläche der verdauenden Wirkung der Magensäfte nicht widerstehen kann, schützt er sich durch einen dicken schleimigen Überzug, der sich über eine grofse Anzahl von Drüsenausgängen hinweglegt und so die Ausscheidung der Magensäfte und eine Verdauung der aufgenommenen Nahrung in hohem Malse hemmt. Die medizinische Behandlung ist durch die Lebensgewohnheiten des Wurmes aufserordentlich erschwert, da infolge der Schwerkraft nur voluminöse Medikamente auf die, an der oberen Magenseite festsitzenden Parasiten einwirken können. Ein weiteres Hemmnis stellt die Schleimschicht dar, die zunächst gelöst werden mufs, bevor ein Gift, auf den Wurm selbst einwirken kann. Von günstigem Erfolg ist die von Hutcheon empfohlene Methode, zunächst eine Dosis Paraffinöl einzugeben, die zur Vergröfserung des Volumens zu gleichen Teilen mit Milch gemisch wird. 1:1 1. pro erwachsenen Vogel bedeutet ein ausreichendes Quantum. Das spezifisch leichtere Öl wird im Magen auf der Milch schwimmen und lösend auf die Schleimschicht einwirken können. Ist auf diese Weise der Weg za den Würmern freigemacht, so wird nach $z$ wei bis dreitägiger Ruhepause eine Mischung von Terpentin, Karbolsäure und warmem Wasser im Verhältnis von 1:1:25 eingegeben, wodurch die Würmer sofort abgetötet werden. $\mathrm{Da}$ die Terpentin-Karbolsäuregabe, ebenso wie das Paraffin, anf nüchternen Magen verabreicht werden mufs, dieser Zustand jedoch jeweils ein 18 stündiges Fasten verlangt, aufserdem der Magen durch die scharfen Gifte sehr schwächt zu werden pllegt, kommen die Tiere bei einer solchen Radikalkur stets in ihrer Kondition derart herunter, dafs sie nur sehr minderwertige Federn zu produzieren vermögen und sich erst nach mehreren Monaten völlig erholen.

Jeder Straufsenfarmbetrieb mufs neben der Erzielung einer fehlerfreien Feder auf die Vermehrung seiner Herde aus dem eigenen Bestande bedacht sein und wird bei der Handhabung seines Wirtschaftsbetriebes auch denjenigen Faktoren, welche die Qualität seiner $\mathrm{Nachzu} \mathrm{cht}$ beeinflussen, eindringlich Rechnung tragen.

Vor allen Dingen sind hierbei die Prinzipien der Zuchtwahl, deren schon weiter oben Erwähnung getan wurde, zu berïcksichtigen, wobei ein peinliches Einhalten dieser Grundsätze eine 
gewisse Garantie dafür bietet, das die Zuchtergebnisse befriedigende sind. Um den Gesundheitszustand der Herde und die Qualität der Zucht- und Federvögel stets anf der wünschenswerten Höhe zu erhalten, ist allzu ausgeprägte Inzucht $\mathrm{zu}$ vermeiden. Während Herdeninzucht in den ersten Generationen zuchtverbessernde Wirkung ausüben kann, zeigen sich in den späteren Generationen Degenerationsmerkmale, die sich in extremen Federnbildungen oder starker Veranlagung 2u Krankheiten äufsern. Fine Blutaufirischung durch angekaufte Zuchthähne ist daher unbedingt erforderlich.

Besonderer Nachdruck muls ferner darauf gelegt werden, dals die Elterntiere zur Zeit der Paarungsperiode sich in guter, kräftiger Konstitution befinden, da es wissenschaftlich nachgewiesen ist, dafs der Gesundheitszustand der Eltern im Augenblicke der Keimbefruchtung ausschlaggebend für die Konstitution der jungen Tiere ist. Da die Brunstperiode zu einer Zeit einsetzt, in welcher nach monatelanger Trockenheit die natürlichen Futtermittel knapp geworden sind, das verdorrte Weidefeld nur noch geringen Nährwert besitzt, muls auch in extensiven Betrieben eine Kraftfuttergabe erfolgen. In solchen Wirtschaften, die grofses Gewicht auf die Erzeugung einer grofsen Menge von jungen Straufsen legen, die also neben dem Ersatz und der Vermehrung des eigenen Herdenbestandes den Verkauf von Zuchttieren betreiben, kann durch Verabreichung anregenden Körnerfutters die Paarungs- und Legetätigkeit der Straufsen erhöht werden. Diese vermehrte Eierproduktion kann jedoch nur durch;die Methode der Inkubatorenbrütung ausgenutzt werden.

Durch praktische Verbesserungen wurden die Brutapparate zu einer derartigen Vervollkommnung gebracht, dafs vom technischen Standpunkte aus betrachtet die Brut durch den Inkubator derjenigen durch die Eltern völlig gleichwertig ist. Welche Methode daher zur Anwendung kommen wird, mus von den wirtschaftlichen Verhälinissen, welche die Betriebsrichtung beeinflussen, abhängig gemacht werden. Bei der natürlichen Brutm ethode sind folgende Momente in Betracht zu ziehen: Als die naturgemälsere hat sie mit geriugerem Risiko $z u$ rechnen. Da der gröfste Teil der Sorge um die Eier den Fiternvögeln überlassen wird, bedeutet sie eine erhebliche Entlastung des Betriebsleiters verbunden mit einer nicht $2 u$ unterschätzenden Vereinfachung und infolgedessen Verbilligung des gesamten Betriebes. Auf der anderen Seite werden jedoch die Straufse durch die stetige Inanspruchnahme durch das Brutgeschäft in hohem Mafse am Eierlegen gehindert, durch die Beschränkung auf 3-4 Gelege zu je 12-16 Eiern wird die Gesamtproduktionsfähigkeit an jungen Straulsen erheblich reduziert. Schliefslich werden die Federn durch die sitzende Bescbäftigung der brütenden Vögel erheblich gefäbrdet, so dafs stets eine gewisse Fntwertung des Federnmaterials in Kauf genommen werden muls. Der Brutapparat 
dagegen versetzt uns in die Lage, diese zum Teil schwerwiegen den Nachteile zu vermeiden. Die Eierproduktion und somit die Anzahl der Kücken kann in ganz vorzüglicher Weise vermehrt werden, da von einer Henne im Durchschnitt 120-140 Eier pro Paarungsperiode erwartet und zum Ausschlüpfen gebracht werden können. Aufserdem ist die Kontrolle über die Zuchtwahl in weitaus höherem Mafse möglich, als dies die natürliche Bebrütung gestattet. Sämtliche, von einem Hahn befruchteten Eier werden in gesonderten Apparaten ausgebrütet, wodurch eine Identifizierung der Jungen ermöglicht wird, und daher bei genauer Beobachtung ein zutreffendes Urteil über die Art und Stärke der väterlichen Individualpotenz gebildet werden kann. Die Verwendung der Brutmaschine verlangt jedoch anderseits eine absolut genaue Kontrolle, unablässige Aufsicht und vorsichtigste Sorgfalt, wenn sie dauernden Erfolg versprechen soll. Das Risiko dieser Methode liegt darin, dafs der geringste Verstofs gegen die auf eingehenden Forschungen basierenden Vorschriften den Verlust sämtlicher im Apparate befindlicher Eier zur Folge haben kann.

In extensiven Betrieben, welche auf eine möglichste Beschränkung der zur Erzielung eines relativ guten Produktes aufgewandten Mittel an Geld und Arbeitskraft Wert legen müssen, wird daher die natürliche Bebrütungsmethode angebracht sein. Intensive Wirtschaften dagegen, in welchen die wirtschaftlichen Faktoren eine rationelle Ausnutzung sämtlicher Produktionsmöglichkeiten verlangen und daher hohe Qualitätszucht sowobl in Federn als auch in Verkaufstieren betrieben werden muls, können ohne Verwendung des Brutapparates eine Rente nicht abwerfen.

Die übergrofse Empfindlichkeit der Jungen in den drei ersten Monaten ihres Daseins bedingt eine grofse Sorgfalt in der Kückenaufzucht. Mangelnde Pflege in diesem jugendlichen Stadium wird an sich günstig veranlagte Tierchen schädigen und solche, in deren Organismus konstitutionelle Neigung zu Krankheiten vorbanden ist, die bei guter Pflege überwunden werden könnte, völlig entwerten. Vorzüglich ist darauf $\mathrm{zu}$ achten, dafs die Tierchen genügend Bewegungsfreiheit haben, vor Kälte und Nässe ausgiebig geschützt werden und nur bekömmliche und die Verdauung befördernde, laxierende Nahrung erhalten. Um die rasch wachsenden Knochen zu festigen, mufs ihnen regelmäfsig ein gröfseres Quantum Knochenmehl oder gehackter Knochen verabreicht werden, dem eine kleine Menge Schwefelblüten beigefügt werden kann. Trotz bester Haltung wird jedoch stets mit einer recht beträchtlichen Sterblichkeit gerechnet werden müssen. Neben allen erdenklichen äufseren Unfällen, wie Verlusten durch Raubtiere oder sonstigen, nicht vorauszusehenden Zufälligkeiten, welche zum Tode dieser kleinen Tierchen führen können, sind die Kücken bei ihrer zarten Konstitution den Straulsenkrankheiten in ganz besonderem Grade ausgesetzt. Das Auftreten der 
Taenia und des Strongylus ist in den ersten Monaten besonders gefährlich und führt in den meisten Fällen zum Tode des Patienten. Mangelnder Schutz vor nasser Kälte erzeugt vielfach Lungenerkrankungen, die in ihrem akuten Verlaufe ein ärztliches Eingreifen vereiteln und diphteritisähnliche Halsaffektionen, die stellenweise endemisch aufgetreten sind, stellen eine erhebliche Gefahr dar. Besonders schwer sind jedoch die Folgen einer fast ausschliefslich Straulsenkücken in den ersten Lebensmonaten befallenden Krankheit, der sogenannten Gelbleber (jellow liver). Gelbleber stellt nach Hutcheon eine Art Atrophie der Leber dar, mit gleichzeitiger krankhafter Verfettung der Zellen. Die Leber zeigt eine gelbliche Färbung, die Schnittfläche ist glatt, nicht körnig wie in gesundem Zustande; die Muskelfasern sind blafs und weich, Herz und Blutgefälse enthalten helles, wässeriges Blut, so dals alle äufseren Anzeichen auf hochgradige Blutarmut schliefsen lassen. Die Anomalie scheint hervorgerufen durch mangelhafte Verdauung der in grofsen Mengen im Magen angehäuften Futterstoffe. Die Ursache der Krankheit ist mit Bestimmtheit noch nicht nachgewiesen, doch gebührt von den mancherlei Vermutungen der Theorie D. Hutcheons die vornehmlichste Beachtung. Er bält die Gelbleber nicht für eine Infektionskrankheit, sondern sucht ihren Ursprung in der mangelhaften Ausbildung der durch angeborene Schwäche empfindlichen, durch ungenügende Pflege in der Entwickelung gehemmten Verdauungsorgane. Die eigentliche Ursache wäre daher nicht in den betroffenen Tieren selbst, sondern in den eine solche Schwäche vererbenden Elterntieren zu suchen. Hierin scheint ein durch die Domestizierung hervorgerufenes Degenerationsmerkmal zum Ausdruck zu kommen. Wenn auch diese Theorie noch nicht einwandsfrei nachgewiesen ist, scheint mir doch hier der Punkt zu sein, an welchem anfassend die wissenschaftliche Forschung diese überaus wichtige Frage lösen könnte.

Neben diesen die Endprodukte der Straufsen-Zuchten beeinfluss enden Faktoren tragen zur Präzisierung der verschiedenen Betriebsmethoden die wirtschaftlichen Bedingungen, unter deren Einwirkung die betreffende Farm steht, in erheblichem Mafse bei. Je nach der Beschaffenheit des Bodens, des Klimas und der wirtschaftsgeographischen Verhältnisse wird eine $\mathbf{A b}$ stufung von intensivster zu extensivster Arbeitsweise nötig werden, wie sie sich auch tatsächlich in den in Südafrika existierenden Farmbetrieben herausgebildet hat. Trotz mancher verwischender Übergänge und vielerlei Ineinandergreifens lassen sich drei ziemlich scharf getrennte Systeme unterscheiden, deren jeweilige Anwendung und Prägnanz fast ausschliefslich durch die Lage und Eigenart des betreffenden Farmgrundstückes und nur in geringem Mafse durch die Liebhaberei des Züchters bedingt ist.

1. Freiweidebetrieb. Der Freiweidebetrieb stellt die Wirtschaft extensirster Arbeitsweise dar: Sein $\mathrm{Z}$ weck besteht 
darin, auf billigem Boden, bei möglichster Beschränkung des toten Inventares eine möglichst grofse Anzahl von Straufsen unter annähernd natürlichen, und daher nur geringe Pflege und geringen materiellen Aufwand beanspruchenden Lebensbedingungen, nutzbringend in Gefangenschaft zu halten. Grundlegend für eine solche extensive Wirtschaftsweise ist billiger, leicbt zu verzinsender Boden. Der Freiweidebetrieb beschränkt sich daher auf solche Gegenden, welche in wirtschaftlicher und klimatisch-geologischer Hinsicht weniger günstig veranlagt sind. Solche, die Produktionskraft der betreffenden Ländereien hemmende Bedingungen verursachen eine relativ geringe Bevölkerungsdichte, erlauben daher für europäische Begriffe ungewohnte Ausdehnung der einzelnen Besitzungen; wozu allerdings andererseits die mangelhafte Fruchtbarkeit des Bodens den Grundbesitzer nötigt. Die Ernährung der auf solchen Betrieben gehaltenen Straufse basiert auf den durch die natürliche Vegetation dargebotenen Nährstoffen. Im speziellen Falle kommt bauptsächlich die ursprüngliche, engere Heimat der Straufse, die Caroo, sowie die Süfs- und Sauerveldtgegend in Betracht. So zusagend diese Ernährungsweise auch für die Straulse ist, müssen doch bei der geringen Produktionskraft dieser Gegenden zum ausreichenden Unterhalt relativ kleiner Herden grofse Areale zur Verfügung stehen. Durchschnittlich rechnet man 6-8 ha (15-20 acres) mittelguter Steppenweide auf einen Straufsen. Die Gesamtausdehnung der Farmen beträgt 3000 bis 5000 ha, so dafs man eine Bestockungsfähigkeit von 300-500 Vögeln annehmen kann, ohne befürchten zu müssen, daIs in schlechten Jahren, bei übermäfsiger Trockenheit das Weidefutter nicht mehr genügen könnte. Um den Betrieb jedoch einigermafsen von den Launen der Witterung unabhängig zu machen, ist es auch in extensivsten Betrieben ratsam, wo es irgend die Wasserverhältnisse gestatten, Luzerne als Futtermittel anzubauen. Jedenfalls mufs für das Vorhandensein des auch nnter ungünstigen Verbältnissen gedeihenden Feigenkaktus, sowie der gleich anspruchslosen Agave gesorgt werden.

Die Haltung der Straufse wird in der Art geregelt, dafs annähernd gleichaltrige Tiere in Herden zusammengestellt werden, die in getrennten Triften unterzubringen sind. Junge Tiere, kränkelnde, sowie besonders wertvolle Exemplare sind auf frischwachsende Triften zu schicken, während die anderen, weniger ansprucbsvollen Herden zur Nachweide aufgetrieben werden können. Hieraus ergibt sich die Notwendigkeit, das vorhandene Areal durch Einzäunungen in verschiedene Schläge aufzuteilen, deren Ausdehnung je nach Güte des Weidefeldes und der Kopfzahl der Herden auf verschiedenen Betrieben wechseln kann. Neben solch gröfseren Abteilungen im durchschnittlichen Umfange von 200-300 ha, werden kleinere Weideplätze für einzelne Paare eingerichtet, wobei ca. 30 ha äuf das Paar gerechnet werden muls. Da das Wasserbefürfnis der Straưse nicht übermälsig

Journ. 2 Orn. LIX. Jahrg. Januar 1911. 
grofs ist, gehört eine Tränkstelle in jede Trift nicht zu den absoluten Erfordernissen, obgleich es natürlich im Interesse der Vereinfachung des Betriebes wünschenswert wäre. $\mathrm{Da}$ die Straufsen in Bezug auf die Art von Futterpflanzen, welche sie zur Nahrung aufnehmen, etwas wählerisch sind, manche Pflanzen gânzlich verschmähen, bei anderen nur die Blätter abstreifen, müssen zur völligen Ausnutzung der Weide gelegentlich Rinder oder Schafe aufgetrieben werden. Hierbei ist noch als günstige Nebenerscheinung aufzuweisen, dafs während dieser Zeit die Möglichkeit besteht, dafs die Eier oder Larven der Parasiten, deren Lebensdauer aufserbalb des Wirttieres wir zwar nicht kennen, aber doch als beschränkt annehmen dürfen, zum Absterben kommen. Die Nachzucht, welche in solchen Betrieben hauptsächlich zur Mehrung des eigenen Herdenbestandes und nur in geringen Fällen zum Verkaufe als Zuchtmaterial verwandt werden wird, ist zweckmäfsiger Weise durch natürliche Brut zu erzielen. Der Freiweidebetrieb kaun unter passenden Verhältnissen in seiner Extensität sehr günstige Resultate aufweisen. Allerdings ist streng darauf za halten, dafs das Princip dieser Extensität in der Anlage und Durchführnng des gesamten Betriebes auf jede Weise gewahrt bleibt, damit das Anlagekapital, welches durch den Erlös an Straufsenfedern zu verzinsen ist, durch keinerlei irgendwie vermeidliche Ausgaben erhöht werde. Totes Inventar, sowie die auf den einzelnen Straufsen aufgewandte Arbeit ist auf ein Minimum zu beschränken. Die Sorge für das einzelne Tier mufs auf die ersten drei Monaten konzentriert werden, da sich jegliche, für die Kückenaufzucht aufgewandte Arbeit rentieren wird, während nach dieser Zeit die Herden mehr und mehr sich selbst überlassen bleiben müssen. Diese Art der Haltung verlangt zwar nur geringe Pflege, verursacht daher wenige Kosten, muls jedoch infolgedessen mehr auf die Quantität als auf die Qualität der zu liefernden Produkte sehen.

2. Bruth of irtschaft. Anders liegen die Verhältnisse bei der Bruthofwirtschaft, deren Existenzbedingung in der rentabeln Verzinsung hohen Bodenkapitals beruht. Die hierdurch bedingte, äulserst intensive Wirtschaftsmethode ist jedoch nur unter besonders günstigen klimatischen und wirtschaftsgeographischen Verhältnissen möglich, ein Grund, aus welchem sich das seltene Vorkommen reiner Bruthofwirtschaften erklären lälst. Die hohen Bodenpreise sowie die Besiedelungsdichte solch günstig veranlagter Ländereien erlauben keine allzugrofse Ausdehnung der einzelnen Betriebe. Der Weideauslauf ist daher so beschränkt, dals die natürliche Vegetation bei weitem nicht zum Unterhalt der Straufsen genügt und durch den Anbau von Futterpflanzen ergänzt resp. ersetzt werden mufs. Bruthofwirtschaft setzt demnacht ein gut funktionierendes Bewässerungssystem voraus, das zum Anbau von Luzerne und anderen Futtermitteln unbedingt erforderlich ist. Je nach der Güte des Bodens und der Wasserverhältnisse wird 
die Kapazität eines bestimmten Areals zur Aufnahme von Straufsen schwanken, doch kann man ungefähr 10-15 ausgewachsene Straufse auf 1 ha Luzernenfeldes rechnen. Die verschiedenen Einfriedungen, auf welchen die Straulse geweidet werden, sind daher in ihrer Ausdehnung durch die Fruchtbarkeit des Bodens, die Grölse der Farm und die Höhe der Bestockung bedingt. Neben solchen Luzernentriften, auf welchen das Gros der Herde, die Kücken, die ein-, zwei- und dreijährigen sowie die reinen Federvögel gehalten werden, sind in derartigen $\mathrm{Be}-$ trieben noch besondere Bruthöfe eingerichtet. $\mathrm{Da}$ in Bruthofwirtschaften aus wirtschaftlichen Gründen in der Regel das Inkubatorensystem angewandt wird, besteht die Möglichkeit, jedem Zuchthahne $z$ wei Zuchthennen zuzuteilen, welche dann in kleinen, ca. 1 preufsischen Morgen grolsen Einzäunungen während der Paarungszeit gehalten werden. Diese Höfe weisen einen Schuppen auf, in dessen Nähe der Nistplatz angelegt wird und sind auf einer Seite mit schattenspendenden Bäumen zu bepflanzen, um den häufig aufserordentlich wertvollen Zuchttieren in jeder Hinsicht Schutz vor den Unbilden der Witterung zu bieten.

Die Ernährung der Tiere erfolgt teils durch Weidegang auf Luzerne, teils durch Verabreichung von Grünfutter oder Luzernenbäcksel. Den Brutvögeln wird eine regelmälsige Ration von Körnerfutter verabreicht, durch deren Menge es der Züchter in der Hand hat Anfang, Dauer und Ende der Brunstperiode in hohem Mafse zu beeinflussen. Körnerfutter und Luzernenheu auf Vorrat aufgestapelt, machen den Betrieb fast vollkommen unabhängig von den Einflüssen der Witterung. Die Bruthofwirtschaft stellt daher eine absolut intensive Betriebsmethode dar, welche die Straufsen in einen hohen, der europäischen Haustierhaltung ähnlichen Zustand der Domestikation versetzt. Die aufserordentlich sorgfältige Pflege, welche in einer solchen Wirtschaft dem einzelnen Tiere zugewandt werden kann, ermöglicht und verlangt bei rationellem Betriebe eine ausgesprochene, reine Qualitätszucht.

3. Kombinierter Betrieb. Bei weitem am häufigsten wird jedoch ein kombinierter Luzernen- und Freiweide-Betrieb am Platze sein. Manche Gegenden werden in ihren Wasser- und Bodenverhältnissen den Anbau von Futtermitteln nur in beschränktem Mafse zulassen, sodals es angebracht sein wird, neben einigen Luzernenschlägen für ausgiebigen Auslauf auf das freie Feld zu sorgen. Hierdurch wird häufig die Möglichkeit gegeben, die vorhandenen natürlichen Bedingungen vollständig auszunützen, ohne durch allzusehr verteuerte Wirtschaftsweise die Rentabilität einer solchen Anlage in Frage zu stellen. Für derartige Betriebe lassen sich natürlich keine allgemein gültigen Zahlen darüber angeben, wie viele Straufse auf einer bestimmten Fläche gehalten werden können, da je nach den vorliegenden Verhältnissen bald grölsere, bald kleinere Areale für den Anbau von Futterpflanzen 
zur Verfügung stehen. Doch wird 100 Straufse auf 250 ha ungefähr zutreffend genannt werdeu können. Für das Paar ausgewachsener Straufse sollten also annähernd 5 ha guter natürlicher Weide zur Verfügung stehen, auf welcher, bei normalen Regenverhältnissen, einen grofsen Teil des Jahres hindurch ausreichend Nährst offe vorhanden sein werden. Während der Trockenzeit, zu der alle nicht bewässerten Pflanzen einen grofsen Teil ihres Nährwertes einbüfsen, werden die Vögel zweckmäfsig morgens und abends gefüttert und den Tag über auf der natürlichen Weide belassen, während besonders wertvolle Tiere und solche, deren 'Gesundheitszustand kräftige Nährstoffzufuhr verlangt, am besten ganz auf Luzerne gehalten werden. Welche Art der Bebrütungsmethode der Züchter in diesem Betriebe anzuwenden gedenkt, mufs von dem speziellen $\mathrm{Z}$ weck seiner Anlage abhängig gemacht werden. Will er hauptsächlich auf Erzeugung einer guten Feder hinarbeiten, seinen Herdenbestand jedoch durch Zukauf guter Zuchthähne von Zeit zu Zeit aufbessern, so wird im Interesse der Billigkeit und Bequemlichkeit des Betriebes die natürliche Methode angebracht sein. Andererseits aber wird eine gute Nachzucht und der Verkauf rassereiner Zuchtvögel die Mehraufwendungen an Geld und Arbeit, die durch den Inkubatorenbetrieb verursacht werden, vollständig bezahlt machen. Der kombinierte Luzerne- und Freiweidebetrieb wird bei rationeller Wirtschaft stets einen guten Reinertrag abgeben, wenn in ihm das, auch für viele andere Land wirtschaftsbetriebe zutreffende, grundlegende Prinzip der extensiven Organisation und intensiven Arbeit befolgt wird. Auf diese Weise ist es möglich, unter relativ ungünstigen Verhältnissen grofse Quantitäten ziemlich hochwertiger Produkte zu erzielen.

\section{Dentsche Ornithologisehe Gesellsehaft.}

\section{Bericht tiber die September-Sitzung 1910.}

Verhandelt Berlin, Montag den 4. September abends 8 Uhr im Architekten-Vereinshause, Wilhelmstrafse 92.

Anwesend die Herren: v. Versen, K. Neunzig, Haase, Jung, Kracht, Schiller, Krause, Graf v. Zedlitz, 0 . Neumann, Schalow, Reichenow, Deditius und Heinroth.

Als Gäste: Herr Brehm und Frau Hein roth.

Vorsitzender: Herr S c halow, Schriftführer Herr Hein roth.

Die Herren Reichenow und Schalow legen die eingegangenen Schriften und Bücher vor.

Herr Graf v. Zedlitz, der ganz kürzlich von einer Spitzbergenreise zurückgekehrt ist, sodafs also eine genaue Bearbeitung 